\title{
Research on Motion Control of AUV with Hybrid Actuators
}

\author{
Zhang Lei ${ }^{1, a}$, Jiang Da-peng ${ }^{1, b}$, Huang Shu-ling ${ }^{1, c}$ and Zhao Jin-xin ${ }^{1, d}$ \\ ${ }^{1}$ College of Shipbuilding Engineering, Harbin Engineering University, Harbin 150001, China \\ State Key Laboratory of Autonomous Underwater Vehicle, Harbin Engineering University, Harbin \\ 150001, China \\ acCharlei08@163.com, bjiangdapeng@hrbeu.edu.cn, clingqing.2009@163.com \\ dsunny_zhao1984@yahoo.com.cn
}

Keywords: autonomous underwater vehicle; hybrid actuators; motion control; stability

\begin{abstract}
A switch function is presented to smooth control instructions and avoid system vibration during switch operating underwater vehicles with hybrid actuators. And a Modified S-plane Controller (MSC) is proposed by analyzing underwater vehicle's dynamics and taking static force and coupling effects between the longitude velocity and other dimensions into account. Besides the advantages of S controller such as simple structure, MSC can solve the motion control of underwater vehicle at high speed which is difficult to control with S-plane controller. The stability of MSC is analyzed with Lyapunov function. Finally, MSC is applied to the motion control of an autonomous underwater vehicle controlled by rudders and thrusters. The feasibility of MSC is demonstrated by the results of velocity control, yaw control and depth control tests.
\end{abstract}

\section{Introduction}

The autonomous underwater vehicle (AUV) is widely researched and developed in military application and ocean exploration all over the world. No umbilical cable is required for AUV, and no human interfered. AUV has good stealth and intelligence. AUV becomes the representative and challenging subject in the domain of underwater vehicle ${ }^{[1]}$.

State Key Laboratory of Autonomous Underwater Vehicle in Harbin Engineering University led AUV "OID-I", whose executive mechanism system configured with thrusters and rudders. AUV is a strongly nonlinear system with coupling effects among every degree of freedom (DOF) in high speed. Besides, vibration or even worse divergence will happen because of abrupt change during switch operating underwater vehicles with rudders and thrusters. A control strategy based on executive mechanism is presented in this paper. The S-plane Control is proved to be an effective motion control method in fact ${ }^{[2]}$. Because no coupling motion factors are not taken into consideration in high speed motion, slow response is resulted when time integration is applied for fixed offsets, and vibration also happens in vertical direction. Based on the Sliding Mode Control Theory ${ }^{[3]}$, a Modified S-plane Controller (MSC) by analyzing the AUV motion model is proposed to compensate the coupling motion effects for AUV. The method can satisfy the control requirements, and solve the entire problem mentioned above. The stability of MSC is analyzed with Lyapunov function ${ }^{[4]}$.

\section{Control Strategy Based on Executive Mechanism}

\subsection{Executive mechanism for underwater vehicle}

AUV "OID-I" is a shape of prolate cylinder, with length of about $7.8 \mathrm{~m}$ and weight of approximately $6600 \mathrm{~kg}$ in air. The cruise speed is $5 \mathrm{kn}$, and maximum speed is $7 \mathrm{kn}$. Two ducted propellers to be the main thrusters are mounted aft producing thrust of $110 \mathrm{~kg}$. Two tunnel thruster are aligned on the bow and stern respectively, with maximum thrust of $25 \mathrm{~kg}$ for each propeller. Two pairs of elevating rudders are mounted on the bow and stern in horizontal plane. Besides, a rudder is equipped aft to control the heading of AUV. 
MSC method to be described below is applied for "Instruction Solver" model. And the equilibrium strategy is adopted for "Thrust Distribution". Outputs of the former are processed by the latter model. Control instructions such as voltage and rudder angle are obtained on every executive mechanism finally.

\subsection{Control strategy of evaluating rudders}

In the point of economic view, it is reasonable to control the pitch to realize motion of variable depth by operating evaluating rudders when AUV is in high speed. $\Delta z$ is a function written as $\Delta z=f(u, \theta, w, t) . \Delta z$ is the variation of depth; $u$ is longitudinal velocity of underwater; $w$ is velocity of other degrees of freedom; $t$ is a time parameter; and $f$ is a nonlinear function. Because the period tact of control is fixed, and the velocity of other degree of freedom is comparatively small, the function can be simplified as $\Delta z=f(u, \theta)$, which describes the relationship of depth variety, current trimming angle and longitudinal velocity. The trimming angle can be gotten as $\theta_{d}=f^{-1}(u, \Delta z)$. In the experiments, we take the function below

$$
\theta_{d}=-\frac{\mu \Delta z}{\sqrt{u}}
$$

Equation(1) is an empirical formula obtained through multiple tests referencing the steering equation. $\mu$ is a manual parameter. A maximum threshold exists for $\theta_{d}$ considering the maneuverability of AUV. According to the goal and the current trimming angles, a trimming control moment is acquired through control algorithm, and rudder angle is resolved.

\subsection{The smooth method of switching control between thruster and rudder}

The efficiency of tunnel thruster is high in low speed and will be low in high speed, but the efficiency of rudder is just the reverse. To save energy and ensure efficiency, there is a switching control between propeller and rudder. The vertical thrusters are used to control the heave, submergence and trim of AUV in low speed, the elevator rudder is adopted to distribute thrust in high speed, and a thrust distribution switch occurs. The AUV control system will be oscillating or diverging because of the saltation of control instruction in a certain critical speed, such as an instruction is suddenly turned off and meanwhile another instruction is opened. Therefore, the transformation of control instruction should be smooth for every actuator. An interim should exist in switching control between thruster and rudder, and a switching function is selected shown in Fig.1.

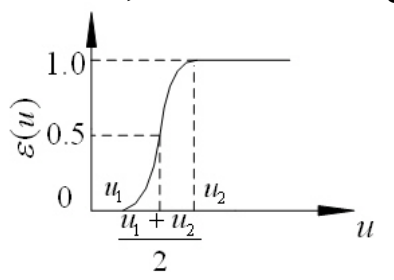

Fig.1 Switching function of force allocation

$$
\varepsilon(u)=\left\{\begin{array}{cc}
0 & 0 \leq u \leq u_{1} \\
0.5+0.5 \sin \frac{\pi}{u_{2}-u_{1}}\left(u-\frac{u_{1}+u_{2}}{2}\right) & u_{1}<u \leq u_{2} \\
1 & u>u_{2}
\end{array} .\right.
$$

$N_{\mathrm{t}}=\varepsilon(u) N, N_{\mathrm{r}}=N-N_{\mathrm{t}}$

$u_{1}, u_{2}$ is obtained according to the relationship between propeller-rudder system efficiency and ship speed. $N_{\mathrm{t}}, N_{\mathrm{r}}$ is the force or torque through normalization produced by thruster and rudder. Fig. 2 shows the response curves with smoothing method and without smoothing method along the 
depth direction when depth is $5 \mathrm{~m}$. It can be seen from the figure that the system oscillation is dramatic decline when the smoothing method is applied.

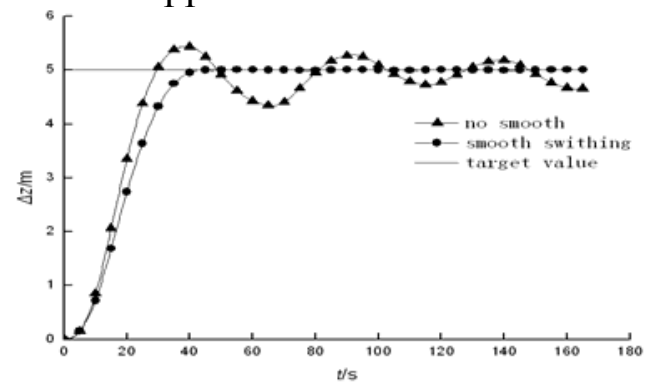

Fig.2 Control simulation test with hybrid actuators

\section{Modified S-plane method based on motion compensation}

\subsection{S-plane controller}

The S-plane controller model can be described as

$$
\Omega=2.0 /\left(1.0+\exp \left(-k_{1} e-k_{2} \dot{e}\right)\right)-1.0+\Delta \Omega \text {. }
$$

Where $e, \dot{e}$ are the control input message (deviation and deviation ratio, processed by normalization) ; $\Delta \Omega$ is the control output, and it denotes the needed thruster of every DOF (after normalization); $k_{1}$ and $k_{2}$ are the corresponding control parameters of deviation and deviation ratio; $\Delta \Omega$ is the disturbing force by self-adaption (normalization). For the ocean current, unknown disturbance or the other factors, the disturbing force over consecutive periods of time is considered, and the offset of S-plane can be adjusted by $\Delta \Omega$ to remove the fixed deviation.

It is known in the test that S-plane has a good control effect when the underwater vehicle worked in a low speed ${ }^{[5-6]}$, but the vehicle is difficult to control with S-plane controller at high speed because the viscous hydrodynamic varying with the velocity, residual buoyancy and restoring moment are ignored. The S-plane controller is only adjusted by adaptive approach, and it will be oscillation and responded slowly in higher residual buoyancy.

\subsection{Modified S-plane method based on motion compensation}

According to the analysis above, a modified S-plane method based on motion compensation is proposed, the control model can written as

$$
\boldsymbol{F}=\hat{\boldsymbol{G}}+\boldsymbol{K}_{s} \exp \left(\alpha u^{2}\right) h(\boldsymbol{s}) .
$$

Where, $\boldsymbol{F}$ is the control output matrix; $\boldsymbol{K}_{s}$ is a positively definite diagonal matrix; $\hat{\boldsymbol{G}}$ is the estimated residual buoyancy and restoring moment; $\alpha$ is a manual parameter, $\alpha>0 . \boldsymbol{s}$ is the same as sliding mode surface in the sliding-mode control, it can be defined as:

$$
\boldsymbol{s}=\Lambda \boldsymbol{T} \tilde{\boldsymbol{\eta}}-\boldsymbol{v}=\boldsymbol{y}-\boldsymbol{v} .
$$

Where, $\boldsymbol{\Lambda}$ is a positively definite diagonal matrix; $\mathrm{T}$ is the transition matrix between hull coordinate system and geodetic coordinate system; $\boldsymbol{\eta}=\left[\begin{array}{lllll}x & y & z & \varphi & \theta\end{array}\right]^{\mathrm{T}}$ denotes the hull position and

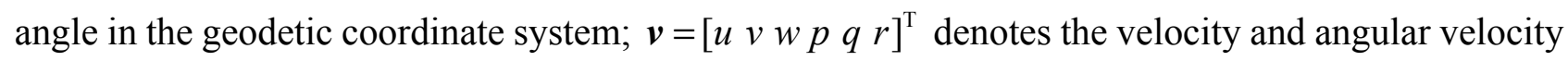
of movement in the hull coordinate system; $\tilde{\boldsymbol{\eta}}=\left[x_{d}-x y_{d}-y z_{d}-z \varphi_{d}-\varphi \theta_{d}-\theta \psi_{d}-\psi\right]^{\mathrm{T}}$ is the deviation of position and angle; The function $h(\cdot)$ can be defined as

$$
h(x)=2.0 /[1.0+\exp (x)]-1.0 .
$$

Curve of $\mathrm{h}(\mathrm{x})$ is S-types. 
It can be conclude that, in the modified S-plane controller, the residual buoyancy and restoring moment of motion model are considered, and the viscous hydrodynamic varying with the velocity is taken into account by a velocity compensate function.

\subsection{Stability analysis}

Because the underwater vehicle is approximately symmetrical about three coordinate planes, The motion model can simplified as ${ }^{[7-8]}$

$$
M \dot{v}+C(v) v+D(v) v+G=T_{v} .
$$

Where, $\boldsymbol{v}=\left[\begin{array}{lllll}u & v & w & p & q\end{array}\right]^{\mathrm{T}}$ denotes the velocity and angular velocity at the six degrees of freedom; $\mathrm{M}$ is the mass matrix, which contains the mass and inertial-mass, and it is a positively definite matrix; $\boldsymbol{C}(v)$ is the inertia force coefficient matrix about velocity; $\boldsymbol{D}(\boldsymbol{v})$ is the viscous hydrodynamic matrix; G denotes the component of gravity and buoyancy at the hull coordinate system; $\boldsymbol{T}_{\boldsymbol{v}}$ is the thrusts and torques at six DOFs produced by actuator.

$$
\begin{gathered}
\boldsymbol{M}=\operatorname{diag}\left[m-X_{\dot{u}}, m-Y_{\dot{v}}, m-Z_{\dot{v},} I_{x}-K_{\dot{p}}, I_{y}-M_{\dot{q}}, I_{z}-N_{\dot{r}}\right] . \\
\boldsymbol{C}(\boldsymbol{V})=\left[\begin{array}{cccccc}
0 & 0 & 0 & 0 & -Z_{\dot{w}} w & Y_{\dot{v}} v \\
0 & 0 & 0 & Z_{\dot{w}} w & 0 & -X_{\dot{u}} u \\
0 & 0 & 0 & -Y_{\dot{v}} v & X_{\dot{u}} u & 0 \\
0 & -Z_{\dot{w}} w & Y_{\dot{v}} v & 0 & -N_{\dot{v}} r & M_{\dot{q}} q \\
Z_{\dot{w}} w & 0 & -X_{\dot{u}} u & N_{\dot{r}} r & 0 & -K_{\dot{p}} p \\
-Y_{\dot{v}} v & X_{\dot{u}} u & 0 & -M_{\dot{q}} q & K_{\dot{p}} p & 0
\end{array}\right] .
\end{gathered}
$$

Where, $\boldsymbol{D}(\boldsymbol{v})$ is the viscous hydrodynamic coefficient matrix which is obtained by the Taylor expansion of the viscous hydrodynamic at a speed, and it is the increasing function of $\mathrm{u}$. $X_{\dot{u}}$ and so on are inertia hydrodynamic coefficients. Important relational expressions in Equation (10) can be obtained after simplification.

$$
\dot{\boldsymbol{M}}=\mathbf{0}, \quad \boldsymbol{C}+\boldsymbol{C}^{\mathrm{T}}=\mathbf{0}, \quad \boldsymbol{D}=g(u)>\mathbf{0} .
$$

Where, $\boldsymbol{C}$ and $\boldsymbol{D}$ are the simple representation of $\boldsymbol{C}(\boldsymbol{v}), \boldsymbol{D}(\boldsymbol{v}) . \boldsymbol{D}$ is a positively definite matrix. A Lyapunov function showed below is selected.

$$
V=\frac{1}{2} \boldsymbol{s}^{\mathrm{T}} \boldsymbol{M s} .
$$

The function above is satisfied with Lyapunov function because $\mathrm{M}$ is a positively definite matrix. Equation (11) can be written as follows after derivation.

$$
\dot{V}=\frac{1}{2} \boldsymbol{s}^{\mathrm{T}} \dot{\boldsymbol{M}} \boldsymbol{s}+\boldsymbol{s}^{\mathrm{T}} \boldsymbol{M} \dot{\boldsymbol{s}} .
$$

According to Equation(10), we can obtain

$$
\dot{V}=-\boldsymbol{s}^{\mathrm{T}} \boldsymbol{D} \boldsymbol{s}+\boldsymbol{s}^{\mathrm{T}}[\boldsymbol{M} \dot{y}-\boldsymbol{F}+\boldsymbol{C y}+\boldsymbol{D} \boldsymbol{y}+\boldsymbol{G}] .
$$

Take Equation (4) into account, and the equation above can be described as:

$$
\dot{V}=-\boldsymbol{s}^{\mathrm{T}} \boldsymbol{D} \boldsymbol{s}+\boldsymbol{s}^{\mathrm{T}}\left[\boldsymbol{M y}+\boldsymbol{C} \boldsymbol{y}+\boldsymbol{D} \boldsymbol{y}+\tilde{\boldsymbol{G}}-\boldsymbol{K}_{\boldsymbol{s}} \exp \left(\alpha u^{2}\right) h(\boldsymbol{s})\right] .
$$

Where, $\tilde{\boldsymbol{G}}=\boldsymbol{G}-\hat{\boldsymbol{G}}$, and an inequality can be obtained:

$$
\dot{V} \leq-\lambda_{\min }(\boldsymbol{D})\|\boldsymbol{s}\|^{2}-\lambda_{\min }\left(\boldsymbol{K}_{\boldsymbol{s}}\right) \exp \left(\boldsymbol{o u c ^ { 2 }}\right)\|\boldsymbol{s}\|+\|\boldsymbol{M} \boldsymbol{y}+(\boldsymbol{C}+\boldsymbol{D}) \boldsymbol{y}+\tilde{\boldsymbol{G}}\| \boldsymbol{s} \| .
$$


$\lambda_{\min }$ is the minimum eigenvalue of corresponding matrix.

If $\boldsymbol{K}_{\boldsymbol{s}}$ meets the conditions as follows, the value of $\mathrm{V}$ will less than 0.

$$
\lambda_{\text {min }}\left(\boldsymbol{K}_{s}\right) \geq \frac{\|\boldsymbol{M} \dot{\boldsymbol{y}}+(\boldsymbol{C}+\boldsymbol{D}) y+\tilde{\boldsymbol{G}}\|}{\exp \left(\alpha u^{2}\right)} .
$$

The value of s will be converged to $0 . \boldsymbol{\Lambda T} \tilde{\boldsymbol{\eta}}-\boldsymbol{v}=0$ when $\boldsymbol{s}=0$. Set the value of $\mathrm{V}^{\prime}$ as $V^{\prime}=\frac{1}{2} \tilde{\boldsymbol{\eta}}^{\mathrm{T}} \tilde{\boldsymbol{\eta}}$, and $\dot{V}^{\prime}=\tilde{\boldsymbol{\eta}}^{\mathrm{T}} \dot{\boldsymbol{\eta}}=-\tilde{\boldsymbol{\eta}}^{\mathrm{T}} \dot{\boldsymbol{\eta}}=-\tilde{\boldsymbol{\eta}}^{\mathrm{T}} \boldsymbol{T}^{-1} \boldsymbol{v}=-\tilde{\boldsymbol{\eta}}^{\mathrm{T}} \boldsymbol{T}^{-1} \boldsymbol{\Lambda} \boldsymbol{T} \tilde{\boldsymbol{\eta}}<0$ because $\boldsymbol{T}^{-1} \boldsymbol{v}=\dot{\boldsymbol{\eta}}$. The value of $\tilde{\boldsymbol{\eta}}$ will be converged to 0 , and the system output will be converged to target value.

\section{Experiments and result analysis}

The control system of a under water vehicle named OID-I is showed in Fig.3. According to the vehicle's structure and actuator configuration, four DOFs' control which contain longitudinal direction, vertical direction, heading direction and trim direction are considered. The longitudinal direction control is the velocity control, which input is velocity deviation and longitudinal acceleration. The inputs of the other directions are still position or angle deviations and their rates of change. In the high speed condition, for the longitudinal direction, in order to avoid rapid change of velocity, the S-plane control based on control output accumulation is applied because the couple effects of longitudinal direction with the other directions is small, and the other DOFs employ the Modified S-plane presented. The sliding mode surface is defined as $\boldsymbol{s}=\boldsymbol{k}_{1} \boldsymbol{e}+\boldsymbol{k}_{2} \dot{\boldsymbol{e}}$ to adjust the influence of control deviation and deviation rate. Where, $\boldsymbol{e}=\left(u_{d}-u y_{d}-y z_{d}-\mathrm{z} \varphi_{d}-\varphi \theta_{d}-\theta \psi_{d}-\psi\right)^{\mathrm{T}}$, $\boldsymbol{k}_{1}=\operatorname{diag}\left(k_{11}, k_{12}, k_{13}, k_{14}, k_{15}, k_{16}\right)$, and $\boldsymbol{k}_{2}=\operatorname{diag}\left(k_{21}, k_{22}, k_{23}, k_{24}, k_{25}, k_{26}\right)$. The control model can described as

$$
\left\{\begin{array}{ll}
f_{i}=\sum K_{s i} h\left(s_{i}\right) & (\mathrm{i}=1) \\
f_{i}=\hat{G}_{i}+K_{s i} \exp \left(\alpha_{i} u^{2}\right) h\left(s_{i}\right) & (\mathrm{i}=2 \cdots 6)
\end{array} .\right.
$$

Where, $f_{i}$ denotes the the needed thrust and torque of ever DOF; $s_{i}$ and $\hat{G}_{i}$ are components of the corresponding vector, $K_{s i}$ is the element at Line $i$, Row $j$ in the diagonal matrix $\boldsymbol{K}_{s}$.

The control tests of longitudinal direction, vertical direction and heading direction in the high speed condition are performed. The control parameter is $\mu=-0.025 ; u_{1}=0.5, u_{2}=0.8 ; \mathrm{K}_{\mathrm{s} 1}=100$, $\mathrm{k}_{11}=7.0, \mathrm{k}_{21}=8.0 ; \mathrm{K}_{\mathrm{s} 3}=100, \mathrm{k}_{13}=0.5, \mathrm{k}_{23}=2.2, \hat{G}_{3}=31, \quad \alpha_{3}=0.8 ; \mathrm{K}_{\mathrm{s} 6}=95, \mathrm{k}_{16}=3.0, \mathrm{k}_{26}=2.0$, $\hat{G}_{6}=15, \quad \alpha_{6}=5$. The control time is $0.5 \mathrm{~s}$.

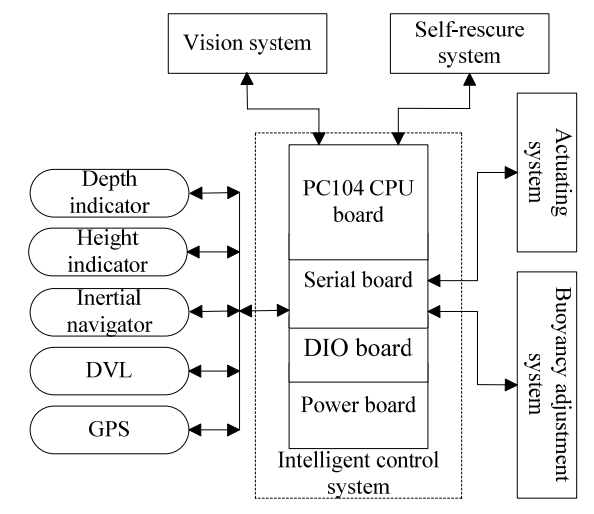

Fig.3 Motion control system specification of AUV

In Fig.4, the target velocity is a sinusoid whose amplitude is $1 \mathrm{~m} / \mathrm{s}$, maximum value is $2 \mathrm{~m} / \mathrm{s}$, and period is $300 \mathrm{~s}$. The heading direction of target is northeast, and the angle is 45 degree; In Fig. 5, the 
target velocity is $2 \mathrm{~m} / \mathrm{s}$, the heading direction is a sinusoid whose amplitude is 45 degree, maximum value is 45 orientated north by east, the period is $300 \mathrm{~s}$; In fig. 6 , the target velocity is a sinusoid whose amplitude is $1 \mathrm{~m} / \mathrm{s}$, maximum value is $2 \mathrm{~m} / \mathrm{s}$, and period is $300 \mathrm{~s}$, the depth of target is $10 \mathrm{~m}$; In Fig.7, the target velocity is $2 \mathrm{~m} / \mathrm{s}$, the target depth a sinusoid whose amplitude is 5 , the maximum value is $10 \mathrm{~m}$, and period is 300s. The tests shown in Fig.4 and Fig.5 are to get the controller performance when longitudinal velocity coupling with heading motion. The tests showed in Fig. 6 and Fig. 7 are to investigate the controller performance when longitudinal velocity is coupled with vertical motion.

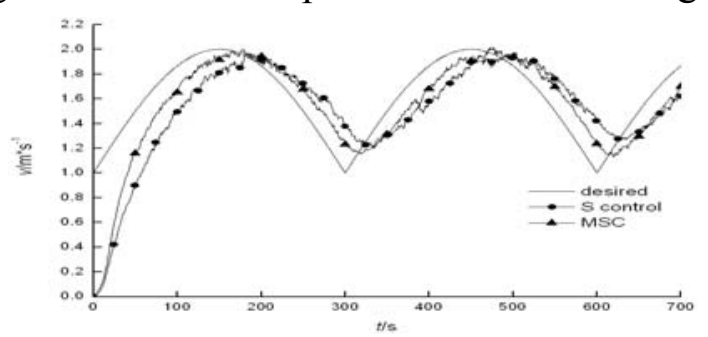

(a)The sinusoidal response of velocity

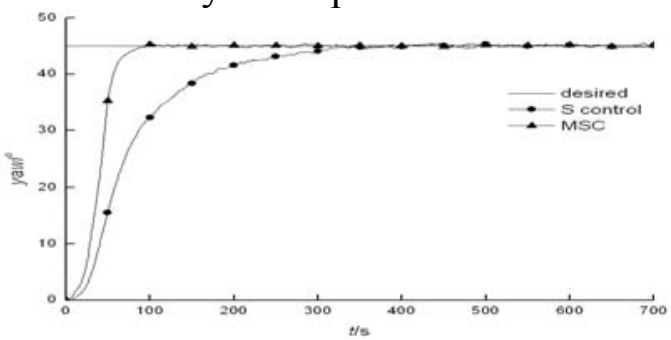

(b)yaw control curve

Fig.4 Sinusoidal response of velocity with yaw control

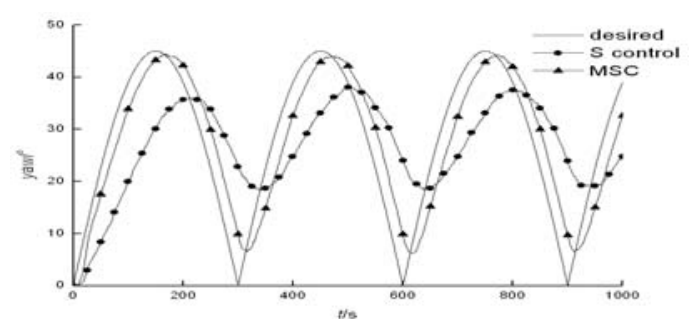

(a)Sinusoidal response of yaw

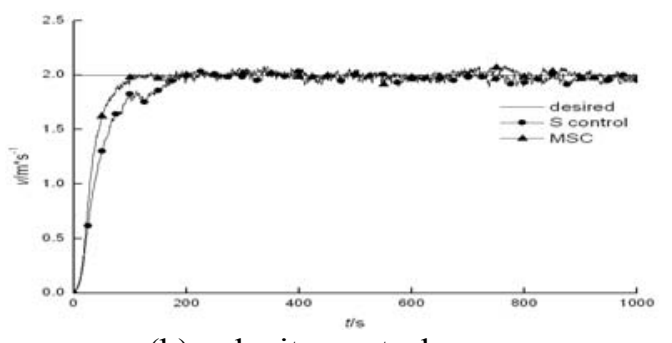

(b) velocity control curve

Fig.5 Sinusoidal response of yaw with velocity control

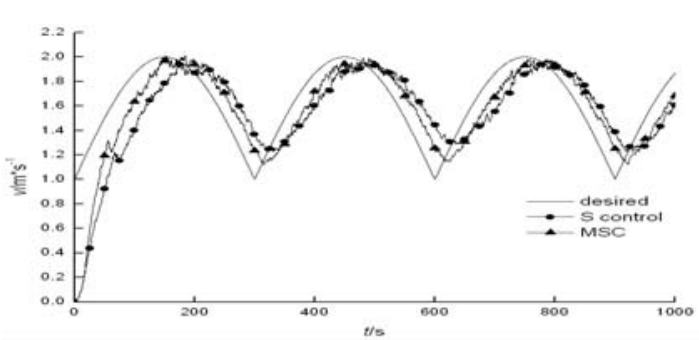

(a) Sinusoidal response of velocity

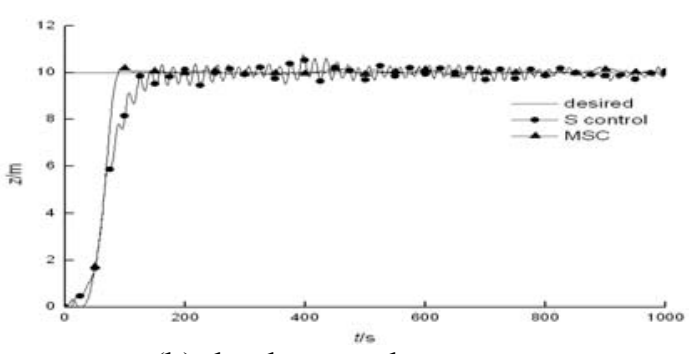

(b) depth control curve

Fig.6 Sinusoidal response of velocity with depth control

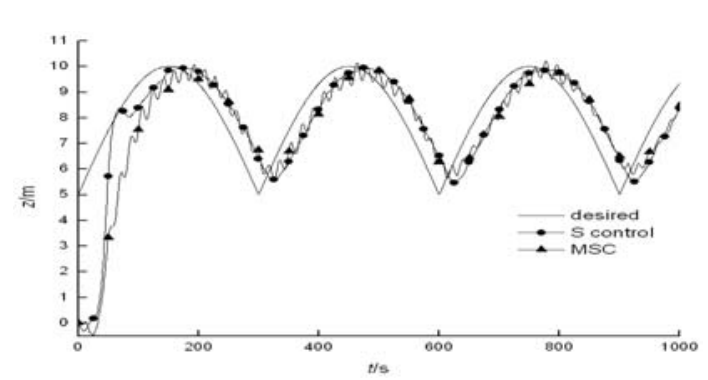

(a)Sinusoidal response of depth

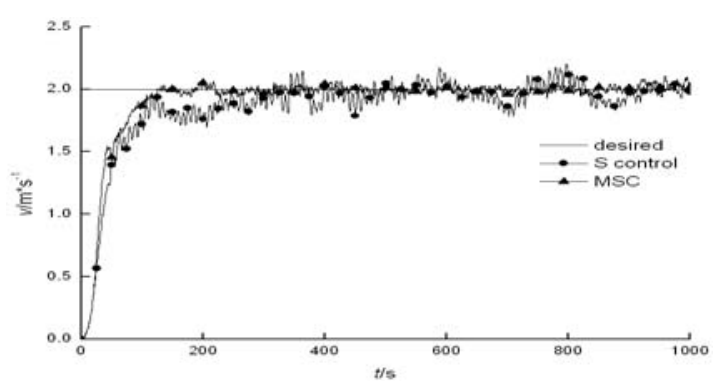

(b)velocity control curve

Fig.7 Sinusoidal response of depth with velocity control

In Fig.4 (a), the modified S-plane controller is faster than S-plane controller in the tracking of velocity variation, but both the controllers didn't reach the velocity base lone of $1 \mathrm{~m} / \mathrm{s}$, and have some several tracking seconds delay in the velocity variation. The reason is that OID-I have a large mass and inertia, especially during the velocity decline period, the thrust deduction is bigger when the propeller is reverse for speed fast decline. Fig.4 (b) showed that the response speed of modified S-plane controller is faster than S-plane controller when considering the couple effects between 
longitudinal motion and heading motion. Fig.5 (a) showed that the modified S-plane controller has a better tracking effect in the high speed condition. In Fig.5(b), with the weak influence of heading motion, both the S-plane controller and modified S-plane controller can achieve stable state for longitudinal motion, but the response speed of modified S-plane is better than S-plane. In Fig.6 (a), with the strong couple effects between lengthways motion and vertical motion, the adaptive ability of modified S-plane controller is better than S-plane in the underwater vehicle motion variation because of motion compensation. In Fig.6 (b) the S-plane controller will be oscillating in the depth control by the influence of longitudinal motion. Fig.7 (a) and Fig.7 (b) denote that the S-plane controller system will be oscillating when longitudinal velocity and depth are controlled at the same time, but the modified S-plane shows a better control effect by considering the motion compensation in the high speed condition.

\section{Conclusion}

The motion control of AUV based on rudders and thrusters is studied in the article. In the vertical plane motion, an approximation function is obtained by the analysis of depth and trim. In the depth control, the function is applied to make the depth project to the trim direction. A switch function is presented to smooth control instructions and avoid system vibration during switch operating underwater vehicles with rudders and thrusters. And a Modified S-plane Controller (MSC) based on motion compensation is proposed. The MSC has the advantages of S controller such as simple structure, easy to adjust parameters, and it can solve the oscillation and slow response for the motion control of underwater vehicle at high speed. The stability of MSC is analyzed with Lyapunov function. The MSC is applied to the motion control of OID-I, and the feasibility of MSC is demonstrated by the results of yaw control and depth control tests at high speed.

\section{Acknowledgment}

This work would not have been possible without important contributions from Li Ye and Wang Zhuo. We gratefully acknowledge the support of the National Natural Science Foundation of China (51209051) and Fundamental Research Foundation of Harbin Engineering University (Grant No. GK2010260119).

\section{References}

[1] XU Yu-ru, PANG Yong-jie, GAN Yong, et al. AUV— — state-of-the-art and prospect [J]. CAAI Transactions on Intelligent Systems, 2006, 1(1): 9-16. (in Chinese)

[2] LIU Xue-min, XU Yu-ru. S control of automatic underwater vehicles [J]. Ocean Engineering, 2001,19(3):81-84. (in Chinese)

[3] TIAN Hong-qi. Sliding mode control theory and application [M]. Wuhan: Wuhan Press, 1995.

[4] HU Shou-song. Autonomous control theory [M]. Peking : National Defense Press, 1994.

[5] GAN Yong, WANG Li-rong, LIU Jian-cheng, et al. The embedded basic motion control system of autonomous underwater vehicle [J]. Robot. 2004, 26(3):246-249. (in Chinese)

[6] GAN Yong. Research on motion control system architecture of underwater vehicle [D]. Harbin: College of Shipbuilding Engineering, Harbin Engineering University, 2007. (in Chinese)

[7] SHI Sheng-da. Maneuverability of Submarine [M]. Peking: National Defense Press, 1995.

[8] CHANG Wen-jun, LIU Jian-cheng, YU Hua-nan et al. AUV mathematical model of Motion Control and Simulation [J]. Ship Engineering, 2002(3):58-60. (in Chinese) 